\title{
BANACH ALGEBRAS OF OPERATORS ON LOCALLY CONVEX SPACES
}

\author{
BY ROBERT T. MOORE ${ }^{1}$
}

Communicated by $\mathrm{H}$. Helson, July 23, 1968

1. Introduction. This is the first of three announcements of results concerning operator theory on locally convex topological vector spaces [3], [4]. The method described here uses certain well-behaved "finite" operators on such a space to study the properties of general operators acting there. Under suitable completeness conditions, these well-behaved operators appear in certain geometrically-determined Banach algebras; this fact brings to bear the highly developed classical theory of Banach algebras in the study of general operators on general locally convex spaces. Some of the methods are formally identical to those introduced by Allan in his earlier spectral theory for locally convex algebras [1].

Two principal applications have motivated the early research reported here and other work now in progress. It would be useful to have an operator theory on locally convex spaces of test functions and distributions powerful enough to treat problems in partial differential and "integro-differential" equations systematically within the framework of functional analysis. The infinite-dimensional representation theory of Lie groups presents a related set of problems, when treated from a systematically "infinitesimal" point of view, on special locally convex spaces of " $C^{\infty}$ vectors." The simplest sorts of applications of this type are illustrated in [4].

Full proofs of these and related results will appear in [5].

2. Calibrations and normed algebras. The notions and results discussed in this section depend upon the choice of a fixed "geometrical" structure on a Hausdorff locally convex topological vector space (lcs) $\mathfrak{X}$ over the ground field $F=\{\boldsymbol{R}$ or $\boldsymbol{C}\}$ : a calibration $\Gamma$ for $\mathfrak{X}$ is a collection of continuous seminorms $p$ on $X$ which induces the topology of $\mathfrak{X}$. Then the collection of all

$$
N(\epsilon, p)=\{u \in \mathfrak{X} \mid p(u) \leqq \epsilon\} \quad \epsilon>0, \quad p \in \Gamma
$$

forms a neighborhood subbase at 0 .

${ }^{1}$ Research supported in part by NSF GP 5585. 
Definition 1. Let $\Gamma$ calibrate a lcs $\mathfrak{X}$ and suppose $T: \mathfrak{X} \rightarrow \mathfrak{X}$ is linear.

(a) Suppose that there exists a finite universal bound $M<\infty$ such that for all $u \in \mathfrak{X}$ and $p \in \Gamma$

$$
P(T u) \leqq M p(u) .
$$

Then $T$ is $\Gamma$-finite, or $T \in \mathcal{F}_{\Gamma}(\mathfrak{X})$, and

$$
\|T\|_{\Gamma}=\sup \{p(T u) \mid u \in \mathfrak{X}, p \in \Gamma, \text { and } p(u)=1\} .
$$

(b) If $\|T\|_{\Gamma} \leqq 1, T$ is a $\Gamma$-contraction, or $T \in \mathcal{C}_{\Gamma}(\mathfrak{X})$.

(c) If $T$ is a bijection, and if for all $u \in \mathfrak{X}$ and $p \in \Gamma$

$$
p(T u)=p(u),
$$

then $T$ is a $\Gamma$-symmetry (or $\Gamma$-rotation) or $T \in \mathbb{R}_{\Gamma}(\mathfrak{X})$.

THEOREM 1. (a) ( $\left.\mathfrak{F}_{\Gamma}(\mathfrak{X}),\|\cdot\|_{\Gamma}\right)$ is a normed algebra with identity over $F$, contained entirely within the algebra $\mathfrak{L}(\mathfrak{X})$ of continuous endomorphisms of $\mathfrak{X}$.

(b) The $\|\cdot\|_{\Gamma-t o p o l o g y}$ on $\mathfrak{F}_{\Gamma}(\mathfrak{X})$ is finer than any inherited topology of $\mathfrak{S}$-convergence ${ }^{2}$ on $\mathfrak{F}_{\Gamma}(\mathfrak{X})$.

(c) The unit ball $\mathfrak{C}_{\Gamma}(\mathfrak{X})$ is a simply closed (in $\mathfrak{X}^{\mathfrak{x}}$ ) convex, balanced, equicontinuous semigroup of operators on $\mathfrak{X}$ (containing the identity $I$ on $\mathfrak{X})$.

(d) $\mathfrak{R}_{\Gamma}(\mathfrak{X})$ is the largest group contained in $\mathfrak{C}_{\Gamma}(\mathfrak{X})$, and lies on the surface of $\mathfrak{C}_{\Gamma}(\mathfrak{X})$.

(e) The action map $(T, u) \rightarrow T u$, the product map $(S, T) \rightarrow S T$ and the inversion map $T \rightarrow T^{-1}$ are continuous, respectively from $\mathfrak{F}_{\Gamma}(\mathfrak{X}) \times \mathfrak{X}$ into $\mathfrak{X}$, from $\mathfrak{F}_{\Gamma}(\mathfrak{X}) \times \mathfrak{F}_{\Gamma}(\mathfrak{X})$ into $\mathcal{F}_{\Gamma}(\mathfrak{X})$, and from the group $\mathcal{G}_{\Gamma}(\mathfrak{X})$ of elements $T$ invertible in $\mathfrak{F}_{\Gamma}(\mathfrak{X})$ into $\mathcal{G}_{\Gamma}(\mathfrak{X})$.

The proof is routine and in large part classical. Notice that if $\mathfrak{X}$ is a normed space, so that $\Gamma=\{\|\cdot\|\}$, then $\mathcal{F}_{\Gamma}(\mathfrak{X})=\mathscr{L}(\mathfrak{X})$ and $\|\cdot\|_{r}$ becomes the usual operator norm. The nonclassical situation presents some novelties, however.

THEOREM 2. Suppose $\mathcal{F}_{\Gamma}(\mathfrak{X})$ is large enough to contain the ideal $\mathfrak{F} R(\mathfrak{X})$ of continuous operators $T$ of finite rank ( $T \mathfrak{X}$ finite-dimensional). Then $\mathfrak{X}$ is normable (admits a calibration $\Gamma^{\prime}=\{\|\cdot\|\}$ ).

Recalling that $\Im \mathfrak{R}(\mathfrak{X})$ is simply dense in $\mathscr{L}(\mathfrak{X})$ ("large") this result says that $\mathfrak{F}_{\Gamma}(\mathfrak{X})$ is "large" iff $\mathfrak{X}$ is normable. The proof uses Theorem 1 (e) and a result of Williamson [6].

${ }^{2}$ Here $\subseteq$ is a family of bounded sets with $\operatorname{span}(U S)=\mathfrak{X}$, and we refer to the topology of uniform convergence on these sets. 
Finally, let $B$ be a closed, convex, balanced, bounded (ccbb) set in $\mathfrak{X}$, let $\mathfrak{X}_{B}=\operatorname{span}(B)$, and for $u \in \mathfrak{X}_{B}$, let

$$
\|u\|_{B}=\inf \left\{\lambda>0 \mid \lambda^{-1} u \in B\right\} .
$$

Then $\left(\mathfrak{X}_{B},\|\cdot\|_{B}\right)$ is a normed space with unit ball $B$ and a finer topology than the relative initial topology from $\mathfrak{X}$. We call $\mathfrak{X}$ normcomplete ("complete in the sense of Mackey") iff every $\left(\mathfrak{X}_{B},\|\cdot\|_{B}\right)$ is complete, as $B$ ranges over the ccbb sets. If $\mathfrak{X}$ is complete, quasicomplete, or sequentially complete, it is normcomplete (see [2]).

Theorem 3. (a) If $\mathfrak{X}$ is normcomplete, then $\mathcal{F}_{\Gamma}(\mathfrak{X})$ is complete, and is a Banach algebra.

(b) Conversely, if $\mathcal{F}_{\Gamma}(\mathfrak{X})$ is a Banach algebra for every calibration $\Gamma$ for $\mathfrak{X}$, then $\mathfrak{X}$ is normcomplete.

This result is essentially a corollary of Theorems 5 and 6 in [2], although a much more transparent proof will be given in [5].

2. Finite operators and recalibration. The following result is the keystone of the theory announced here. It not only leads to all subsequent results, but it also provides the principal bridge between the theory and its applications. Essentially, it removes all dependence upon a fixed "geometrical" $\Gamma$ and places the theory in its proper topological context.

TheOREM 4. (a) A semigroup $\mathfrak{S} \subset \mathfrak{L}(\mathfrak{X})$ is equicontinuous on $\mathfrak{X}$ iff there exists a calibration for $\mathfrak{X}$ such that $\mathcal{S}$ consists of $\Gamma$-contractions: $S \subset \mathfrak{e}_{\Gamma}(\mathfrak{X})$.

(b) Similarly, a group $\mathfrak{S \subset} \mathfrak{L}(\mathfrak{X})$ is equicontinuous iff there exists a $\Gamma$ such that $\mathcal{G} \subset \mathbb{R}_{\Gamma}(\mathfrak{X})$.

(c) Indeed, suppose $\Gamma^{\prime}$ is some given calibration for $\mathfrak{X}$. Then a suitable calibration for (a) or (b) can be constructed by defining for all $u \in \mathfrak{X}$ and $p^{\prime} \in \Gamma^{\prime}$

$$
p(u)=\sup \left\{p^{\prime}(T u) \mid T \in \mathrm{S} \text { or } T=I\right\}
$$

(take $S=\mathcal{G}$ in (b)) and $\Gamma=\left\{p \mid p^{\prime} \in \Gamma^{\prime}\right\}$.

Both the recipe (5) and the proof itself hark back to the well-known classical proof that a Banach space can be equivalently renormed so as to make an operator-norm-bounded semigroup $S$ into a contraction semigroup. The result then focuses our attention upon the following canonical set of operators.

DEFINITION 2. An operator $T \in \mathscr{L}(\mathfrak{X})$ is finite, or $T \in \mathcal{F}(\mathfrak{X})$, iff for some finite $t<\infty, t^{-1} T$ generates an equicontinuous semigroup. 
Corollary 5. $\mathfrak{F}(\mathfrak{X})=\bigcup\left\{\mathfrak{F}_{\Gamma}(\mathfrak{X}) \mid \Gamma\right.$ is a calibration for the lcs $\left.\mathfrak{X}\right\}$.

This corollary allows us to identify innumerable nontrivial operators $T \in \mathcal{L}(\mathfrak{X})$ which must appear in $\mathfrak{F}(\mathfrak{X})$, hence in some nontrivial $\mathfrak{F}_{\Gamma}(\mathfrak{X})$.

Proposition 6. Suppose $T \in \mathfrak{L}(\mathfrak{X})$ satisfies a polynomial identity $P(T)=0$, for $P$ some nontrivial polynomial over $F$. Then $T \in \mathcal{F}(\mathfrak{X})$.

Nilpotent operators, with $T^{k}=0$ for some $k$, and idempotent projection operators, with $E^{2}=E$, are typical examples. Operators of finite rank, being essentially matrices, also satisfy a polynomial identity.

THEOREM 7. The set $\mathscr{L B}(\mathfrak{X})$ of locally bounded operators $T$ in $\mathscr{L}(\mathfrak{X})$ (for some zero-neighborhood $N_{T}, T N_{T}$ is bounded) is an ideal in $\mathscr{L}(\mathfrak{X})$ contained entirely within $\mathfrak{F}(\mathfrak{X})$.

Such familiar operators as compact operators $\left(T N_{T}\right.$ compact or precompact), and hence nuclear operators and operators of finite rank, appear in $\mathscr{L} \mathscr{B}(\mathfrak{X})$ and hence in $\mathfrak{F}(\mathfrak{X})$. If $\mathfrak{X}$ is not normable, Theorem 2 and these observations show that $\mathfrak{F}_{\Gamma}(\mathfrak{X})$ depends heavily on the choice of $\Gamma$ : every finite-rank $T \in \mathcal{F} R(\mathfrak{X})$ lies in some $\mathfrak{F}_{\Gamma}(\mathfrak{X})$, but no single $\mathcal{F}_{\Gamma}(\mathfrak{X})$ can accommodate all of $\mathfrak{F} R(\mathfrak{X})$. In fact, some $\mathfrak{F}_{\Gamma}(\mathfrak{X})$ degenerate into the trivial algebra $F I$, so the recalibration part of Theorem 4 effects a genuine qualitative change in the situation, "making room" for operators not previously admitted to $\mathfrak{F}_{\Gamma}(\mathfrak{X})$.

It is also the case that the $\|\cdot\|_{\Gamma}$ topologies vary wildly with $\Gamma$ : an algebra $Q$ is known which is contained in uncountably many different $\mathcal{F}_{\mathbf{r}}(\mathfrak{X})$ and inherits pair-wise distinct $\|\cdot\|_{\Gamma}$ topologies from these. (Recall that if $\mathfrak{X}$ is Banach, then the operator norm topology on $\mathscr{L}(\mathfrak{X})$ is the unique Banach algebra topology for $\mathfrak{L}(\mathfrak{X})$.)

The following consequence of Theorem 4 is very useful in setting up correspondences between continuous " $\Gamma$-symmetry" representations of locally compact groups $G$ (generalized unitary representations) and continuous representations of their measure algebras $M(G)$ or group algebras $L^{1}(G)$. It admits a clumsy generalization to nonbarreled spaces.

THEOREM 8. Let $\rho$ be a weakly continuous representation of a normed algebra $a$ on a barreled lcs $\mathfrak{X}$. (That is, let $\rho: A \rightarrow \mathfrak{L}(\mathfrak{X})$ be a homomorphism such that for all $u \in \mathfrak{X}$ and $u_{*} \in \mathfrak{X}^{*}, x \rightarrow\left\langle\rho(x) u, u_{*}\right\rangle$ is continuous.) Then there exists a calibration $\Gamma$, such that $\rho$ is a norm-decreasing homomorphism of $A$ into $F_{\Gamma}(\mathfrak{X})$.

Similarly, one can prove a maximality result concerning the $\mathcal{F}_{\Gamma}(\mathfrak{X})$. 
Proposition 9. Suppose $a \subset \mathfrak{L}(\mathfrak{X})$ admits a multiplicative norm $\|\cdot\|$ and norm topology such that $a$ acts continuously on $x$. Then there exists a calibration $\Gamma$ such that $a \subset \mathcal{F}_{\Gamma}(\mathfrak{X})$ and for all $T \in Q,\|T\| \geqq\|T\|_{\Gamma}$.

4. Spectral theory. Let the ground field $\boldsymbol{F}=\boldsymbol{C}$ in this section, write $C^{*}=\mathbf{C} \cup\{\infty\}$, the Riemann sphere, and the usual conventions about holomorphy "at $\infty$ " will hold.

Definition 3. Let $A$ be closed and densely defined on $\mathfrak{X}$.

(a) Then $\lambda \in \boldsymbol{C}$ is in the finite resolvent set $\rho_{F}^{*}(A)$ iff $\lambda-A$ possesses an inverse $(\lambda-A)^{-1} \in \mathcal{F}(\mathfrak{X})$. The point $\infty$ is in $\rho_{F}^{*}(A)$ iff $A \in \mathfrak{F}(\mathfrak{X})$.

(b) The finite spectrum is $\sigma_{F}^{*}(A)=\mathrm{C}^{*} \sim \rho_{F}^{*}(A)$.

ThEOREM 10. Let $\mathfrak{X}$ be normcomplete.

(a) Then $\rho_{F}^{*}(A)$ is open, so $\sigma_{F}^{*}(A)$ is compact in $C^{*}$.

(b) The map $\lambda \rightarrow(\lambda-A)^{-1}$ admits a boundedly convergent power series expansion about each $\lambda \in \rho_{F}^{*}(A)$, so for $u \in \mathfrak{X}$ and $u_{*} \in \mathfrak{X}^{*}$, the function $\lambda \rightarrow\left\langle(\lambda-A)^{-1} u, u_{*}\right\rangle$ is holomorphic.

(c) Suppose $X$ is barreled, and suppose $\lambda_{0}$ is a point in the classical resolvent set $\rho_{c}(A)=\left\{\lambda \in c \mid(\lambda-A)^{-1}\right.$ exists in $\left.\mathfrak{L}(\mathfrak{X})\right\}$ with a neighborhood $N\left(\lambda_{0}\right) \subset \rho_{c}(A)$ such that $\lambda \rightarrow\left\langle(\lambda-A)^{-1} u, u_{*}\right\rangle$ is holomorphic on $N\left(\lambda_{0}\right)$ for all $u \in \mathfrak{X}$ and $u_{*} \in \mathfrak{X}^{*}$. Then $\lambda_{0} \in \rho_{F}^{*}(A)$.

Definition 4. Let $T \in \mathfrak{F}(\mathfrak{X})$. Then the radius of equicontinuity of $T$ is $r_{E}(T)=\inf \left\{t \in(0, \infty) \mid t^{-1} T\right.$ generates an equicontinuous semigroup $\}$.

THEOREM 11. (a) On any commutative algebra $a \subset \mathcal{F}(\mathfrak{X}), r_{B}$ is a multiplicative seminorm such that $r_{E}\left(T^{k}\right)=r_{E}(T)^{k}$ for all integers $k \geqq 0$.

(b) For $T \in \mathcal{F}(\mathfrak{X}), r_{E}(T)$ is the spectral radius

$$
r_{B}(T)=\sup \left\{|\lambda| \mid \lambda \in \sigma_{F}(T)\right\} .
$$

Thus $r_{E}$ exactly imitates the spectral radius for a Banach algebra, where $r_{\sigma}(T)=\lim \left\{\| T^{k}|1 / k| k \rightarrow \infty\right\}$. The proofs are reduced to the Banach algebra theory via an approximation lemma. If $\Gamma$ calibrates $\mathfrak{X}$, write $\sigma_{\Gamma}^{*}(A)=\left\{\lambda \in C^{*} \mid\left[\lambda=\infty\right.\right.$ and $\left.A \in \mathcal{F}_{\Gamma}(\mathfrak{X})\right]$ or $\left[(\lambda-A)^{-1}\right.$ exists in $\left.\left.\mathcal{F}_{\Gamma}(\mathfrak{X})\right]\right\}$ and $\sigma_{\Gamma}^{*}(A)=C^{*} \sim \rho_{\Gamma}(A)$.

Lemma 12. Suppose $U$ is an open neighborhood of $\sigma_{F}^{*}(A)$. Then there exists a calibration $\mathrm{\Gamma}$ such that

$$
\sigma_{F}^{*}(A) \subset \sigma_{\Gamma}^{*}(A) \subset U
$$

This lemma also makes possible an exact generalization of the usual operational calculus for operators $A$ on a Banach space, using the Cauchy integral formula (where it converges). 


$$
f(A)=(2 \pi i)^{-1} \int_{\gamma} f(\lambda)(\lambda-A)^{-1} d \lambda,
$$

for $f$ holomorphic on a neighborhood of $\sigma_{F}^{*}(A)$ and $\boldsymbol{\gamma}$ an arc orientedly encircling $\sigma_{F}^{*}(A)$ inside that neighborhood. The same technique allows an elementary theory of spectral projections associated with compact, relatively open subsets of the spectrum. The methods are nearly those of Allan [1].

\section{REFERENCES}

1. G. R. Allan, A spectral theory for locally convex algebras, Proc. London Math. Soc. 15 (1965), 399-421.

2. R. T. Moore, Completeness, equicontinuity and hypocontinuity in operator algebras, J. Functional Analysis 1 (1967), 419-442.

3. - Adjoints, numerical ranges, and spectra of operators and locally convex spaces, Bull. Amer. Math. Soc. 75 (1969), 85-90.

4. - Generation of equicontinuous semigroups by Hermitian and sectorial operators (in preparation).

5. - Operator theory on locally convex spaces I: Banach algebras, states, and numerical ranges (in preparation).

6. J. H. Williamson, Two conditions equivalent to normability, J. London Math. Soc. 31 (1956), 111-116.

University of California, Berkeley, California 94720 